\title{
Genotypic variation for maize weevil resistance in eastern and southern Africa maize inbred lines
}

\author{
L.C. Kasozi ${ }^{1,2}$, J. Derera ${ }^{2}$, P. Tongoona ${ }^{2}$, P. Tukamuhabwa ${ }^{3}$, A. Muwonge ${ }^{1}$ and G. Asea ${ }^{1}$ \\ ${ }^{1}$ National Crops Resources Research Institute, National Agricultural Research Organization, \\ P. O. Box 7084 Kampala, Uganda \\ ${ }^{2}$ School of Agricultural, Earth and Environmental Sciences, University of KwaZulu-Natal, P. Bag X01, \\ Scottsville 3209, South Africa \\ ${ }^{3}$ Department of Agricultural Production, School of Agricultural Sciences, College of Agricultural and \\ Environmental Sciences, Makerere University, P. O. Box 7062 Kampala, Uganda
}

Author for correspondence: kclwanga@-gmail.com

\begin{abstract}
The maize weevil (Sitophilus zeamais Motschulsky) is the most destructive storage insect pest of maize (Zea mays $\mathbf{L}$.) worldwide, especially among susceptible varieties. Breeding for grain resistance against the weevil is a major component of an integrated pest management strategy in maize production. The objective of this study was to identify diverse sources of weevil resistance for introgression in breeding programmes. A total of 180 inbred lines from three geographical areas were screened for maize weevil resistance. Screening was executed by infesting $50 \mathrm{~g}$ of maize grain with 32 newly emerged adult weevils, placed in $250 \mathrm{~cm}^{3}$ glass jars in a "no-choice" laboratory test. The grain susceptibility parameters used were $F_{1}$ weevil progeny emergence, percent grain damage, median development period, Dobie's index of susceptibility, and parental weevil mortality. New sources of weevil resistance for maize breeding were identified. Eight inbred lines were consistently resistant and, therefore, selected as potential donors for weevil resistance in the maize improvement programmes. There was significant genetic variation, and high levels of heritability $(89-96 \%)$ for weevil resistance that suggested high potential for germplasm improvement through selection. No significant association was observed between maize weevil resistance and grain yield, suggesting that breeding for maize weevil resistance can be achieved without compromising grain yield.
\end{abstract}

Key words: Sitophilus zeamais, weevil resistance, Zea mays

Introduction

The maize weevil (Sitophilus zeamais Motschulsky) is a major storage pest prevalent in all maize growing areas, especially in tropical countries. Weevils usually begin infesting maize grain in the field from where they multiply and cause 
damage up to the processing stage (Demisse et al., 2008). The most economically and environmentally sustainable control measure against weevils, especially at smallholder farmer level, is the use of host plant resistance (Dobie, 1977). Deployment of weevil resistant maize cultivars would greatly minimise losses in grain quantity and quality arising from weevil infestations. However, most of the breeding strategies have been emphasizing other traits, including grain yield enhancement (Tollenaar and Lee, 2006); grain yield and drought stress tolerance (Derera et al., 2007); grain yield, nitrogen, and drought-stress tolerance (Makumbi et al., 2011); and grain yield and resistance to diseases (Vivek et al., 2010). There are only a few studies that have focused on grain improvement for weevil resistance, hence resulting in few weevil resistant varieties available to farmers in tropical environments.

A number of studies have shown that weevil resistance occurs in maize germplasm (Widstrom et al., 1972; Derera et al., 2001; García-Lara et al., 2009; Mwololo et al., 2010). Additionally, results from several studies have revealed the existence of high genetic variability for weevil resistance in a number of maize germplasm (Li et al., 1998; Siwale et al., 2009; Dari et al., 2010), which increases opportunities for maize germplasm enhancement towards weevil resistance. Therefore, identification and utilisation of weevil resistant germplasm lines would be an important step in the development of weevil resistant maize cultivars. Most of the studies conducted on identifying sources of weevil resistance have mainly focused on grain resistance against weevils, but limited attention has been given to a combination of traits (Abebe $e t$ al., 2009; Mwololo et al., 2010). Thus, knowledge of the relationship between weevil resistance and grain yield would be important in the maize improvement strategies against weevil infestation. This is very important to the farmers at the pre and postharvest handling levels of maize production. This study was conducted to determine the genetic variability for weevil resistance in maize inbred lines from eastern and southern Africa regions.

\section{Materials and methods}

\section{Sources and multiplication of maize inbred lines}

For broadening the genetic base for weevil resistance, tropical and sub-tropical maize germplasm was used. The tropical germplasm was obtained from the National Crops Resources Research Institute (NaCRRI), Namulonge, Uganda (51 inbred lines), and the International Maize and Wheat Improvement Centre (CIMMYT), Kenya (55 inbred lines); while the sub-tropical germplasm (74 inbred lines) were sourced from the University of KwaZulu Natal, Republic of South Africa. A total of -180 inbred lines was screened, to which two weevil resistant checks, namely weevil/ CML312]-B-13-2-1-BBB/[weevil/ CML387]-B-9-1-1 (RC 181) and CIMMYT HA1091 (RC 182), and two susceptible checks, namely local popcorn (SC 183) and Longe 5 (SC 184) were added.

The test materials were first planted for seed increase and grain yield estimation in the first and second rainy seasons of 2010. They were planted at NaCRRI (1200 m.a.s.l; $0^{\circ} 32^{\prime} \mathrm{N}, 32^{\circ} 34^{\prime} \mathrm{E} ; 1300 \mathrm{~mm}$ bimodal rainfall). Two rows of $5 \mathrm{~m}$ long were planted per entry in two replications; the first row from which ears for screening 
against the maize weevil were obtained was sib-mated to avoid outcrossing with other genotypes, while the second row was used for estimating grain yield. The inter-row spacing was $0.75 \mathrm{~m}$, while the intra-row spacing was $0.3 \mathrm{~m}$. Diammonium phosphate ( $18 \%$ phosphorus) was applied at planting at a rate of $120 \mathrm{~kg}$ $\mathrm{ha}^{-1}$, while urea ( $46 \%$ nitrogen) was applied 30 days after planting at a rate of $120 \mathrm{~kg} \mathrm{ha}^{-1}$.

\section{Yield estimation}

After drying in the field, the ears were harvested, de-husked and weighed to determine the respective field weight. The ears for each inbred line were then shelled and sampled to obtain about 12 kernels which were used for determining the moisture content. The kernels were then sun dried to a moisture content of $13 \%$ and later prepared for screening against the maize weevil. Grain yield ( $\left.\mathrm{t} \mathrm{ha}^{-1}\right)$ was calculated for each inbred line as follows:

Grain yield $\left(\mathrm{tha}^{-1}\right)=$

\section{((Grain weight (kg/plot))x10x (100-MC) $)$} (100-125) (Plot area)

$\mathrm{x}$ shelling percentage;

Where: $\mathrm{MC}=$ grain moisture content $(\%)$

\section{Weevil rearing}

Prior to the screening exercise, weevils were first multiplied to provide an adequate supply of adult weevils of age 0 to 7 days. This represented the first generation of laboratory reared weevils with known age. Weevil rearing was achieved by obtaining adult weevils from infested maize grain. About 300 unsexed weevils were introduced into $1500 \mathrm{~g}$ of maize grain of variety Longe 5 - (one of the most susceptible maize varieties in Uganda) placed into large plastic jars of volume $3000 \mathrm{~cm}^{3}$.

To provide for proper ventilation, the lids of the plastic jars were perforated and gauze-wire mesh of pore size less than 1 $\mathrm{mm}$ stuck on them (lids) to prevent the weevils from escaping. The weevil-maize culture was incubated for 14 days, in the laboratory, at a temperature of $28 \pm 2{ }^{\circ} \mathrm{C}$ and a relative humidity of $70 \pm 5 \%$, to enhance oviposition. A heat fan and a humidifier were used for regulating the temperature and relative humidity, respectively.

After two weeks, the maize-weevil cultures were sieved using a mesh sieve (Endecotts Ltd, UK), to remove the weevils from the grain. The maize grain was later returned to the plastic jars and incubated under the same conditions, to allow the eggs to hatch and $F_{1}$ weevil progenies to emerge. The test grain was infested using these newly emerged $F_{1}$ progenies of age 0 to 7 days old.

\section{Grain resistance test}

The grain for screening against weevils was first subjected to cold treatment at $20^{\circ} \mathrm{C}$ for 14 days, to get rid of both adult and immature weevils that could have infested the grain in the field. After the cold treatment, the grain was acclimatised to laboratory conditions for seven days under a weevil-free environment and later $50 \mathrm{~g}$ were weighed into glass jars of size $250 \mathrm{~cm}^{3}$. The grain in the glass jars was infested with 32 unsexed adult weevils which were reared as described in the previous section.

The maize grain-weevil cultures were laid out in the laboratory, in a randomised complete block design, with three replications. The cultures were incubated 
for oviposition for 14 days, under controlled laboratory conditions, as described above. After 14 days, the parental weevils were sieved out of the maize grain to ensure that the weevils that subsequently emerged were only $F_{1}$ generation progenies.

Seven days after parental weevil removal, the cultures were monitored on a daily basis to observe for any $F_{1}$ weevil progeny emergence. On first appearance of the $F_{1}$ progenies, the cultures were then monitored every two days for recording and removing any new $F_{1}$ weevil progenies emerging from the grain. This continued until no new $\mathrm{F}_{1}$ weevils emerged from the grain after about 75 days.

\section{Data collection}

Data were recorded on the number of parental weevils alive and parental weevils dead (parental weevil mortality), taken after the oviposition period (14 days). The number of weevils that emerged from each genotype was recorded every after two days, starting from the third week after the weevil-grain culture initiation. The total number of $F_{1}$ weevil progenies that emerged per genotype was obtained by summing-up the total number of weevils recorded per genotype.

The median development period (MDP) of the maize weevil determined for each genotype was calculated as the time (days) from the middle of the oviposition period to the emergence of $50 \%$ of the $\mathrm{F}_{1}$ weevil progenies (Dobie, 1977). Grain damage was calculated as the percentage of damaged grains over total grains per sample. Dobie's index of susceptibility (DIS) was calculated based on the total $F_{1}$ weevil emergence and the median development period for each genotype (Dobie, 1974). It was calculated using the formula:
DIS =

$\log _{\mathrm{e}}$ (No. of $\mathrm{F}_{1}$ weevil progeny emergence)

(Median Development Period)

Where: $\log _{\mathrm{e}}=$ natural logarithms

\section{Data analysis}

The grain and weevil parameters were subjected to the "general linear models" of SAS version 9.1 (SAS-Institute, 2003). The differences between means were detected using the Least Significant Differences at a $5 \%$ probability level $(\mathrm{LSD}=0.05)$. Similarly, the field data on grain yield for the two seasons (2010A and 2010B) were subjected to the same analyses.

Heritability was estimated using genotypic and environmental variance components of the study traits (Hallauer and Miranda, 1988). The variance components were estimated using the REML tool in GenStat version 14 (Payne et al., 2011). During analysis, the genotypes were considered as random effects, while the replications were considered as fixed effects. Heritability was estimated as:

$h^{2}=V g /[(V e / r s)+V g] \times 100$

Where:

$\mathrm{Vg}=$ genotypic variance; $\mathrm{Ve}=$ environmental variance; $r=$ number of replications; $\mathrm{s}=$ number of sites.

Data on inbred line response to weevil infestation using Dobie's index of susceptibility (DIS) were regressed against grain yield to generate scatter plots. 
Categorisation of inbred lines

$\mathrm{F}_{1}$ weevil progeny emergence (FWE), percent grain damage (GD), median development period (MDP), and Dobie's index of susceptibility (DIS) were used to categorise inbred lines into various weevil response classes (resistant, moderately resistant, moderately susceptible, susceptible and highly susceptible). Based on Dobie (1974) index of susceptibility, the 180 inbred lines were allocated into five response classes. The response classes were defined as $1.0-4.0=$ resistant, 4.1 $-7.0=$ moderately resistant, $7.1-10.0=$ moderately susceptible, $10.1-13.0=$ susceptible, and $>13.1=$ highly susceptible.

For the other parameters $\left(F_{1}\right.$ weevil progeny emergence, percent grain damage and median development period), grouping of the inbred lines was based on the response of the resistant and susceptible control check varieties, the least significant differences (LSD) and the trial mean. Inbred lines with data that were not significantly different from the resistant checks were regarded as resistant. Inbred lines with values that were significantly lower than the trial mean but higher than the resistant check were classified as moderately resistant; inbred lines with responses not significantly different from the trial mean were categorized as moderately susceptible. The inbred lines with values that were significantly higher than the trial mean, and those that were not significantly different from the susceptible check were grouped in the susceptible class.

\section{Results}

\section{Genotypic variation}

The mean squares for five grain susceptibility parameters are shown in Table 1 . The mean squares for percent grain damage (GD), Dobie's index of susceptibility (DIS), $F_{1}$ weevil progeny

Table 1. Mean squares for grain susceptibility parameters for the 180 inbred lines and four checks

\begin{tabular}{|c|c|c|c|c|c|c|}
\hline \multirow{2}{*}{$\begin{array}{l}\text { Source of } \\
\text { variations }\end{array}$} & \multirow[t]{2}{*}{$\mathrm{DF}$} & \multicolumn{5}{|c|}{ Mean square } \\
\hline & & FWE & GD & MDP & DIS & PWM \\
\hline Environments & 1 & $40261.68 * * *$ & $191.1 * * *$ & $74.09 *$ & $72.09 * * *$ & 79.90 \\
\hline Replications & 2 & 67910.63 & 1003.58 & 1891.05 & 695.21 & 52.14 \\
\hline Genotype & 183 & $7145.64 * * *$ & $82.38 * * *$ & $203.87 * * *$ & $58.43 * * *$ & 73.30 \\
\hline $\mathrm{GxE}$ & 183 & 677.96 & $6.06 * * *$ & 11.96 & $2.32 *$ & 69.17 \\
\hline Error & 734 & 701.68 & 4.29 & 13.74 & 1.88 & 26.32 \\
\hline $\mathrm{R}^{2}$ & & 0.76 & 0.85 & 0.81 & 0.90 & 0.58 \\
\hline $\mathrm{CV}(\%)$ & & 40.20 & 12.61 & 9.82 & 12.65 & 51.08 \\
\hline
\end{tabular}

$*, * *, * * *$ indicate the value is significant at $\mathrm{P}<0.05, \mathrm{P}<0.01$ and $\mathrm{P}<0.001$, respectively; and ns indicate not significant at $5 \%$ probability level; $\mathrm{FWE}=\mathrm{F}_{1}$ weevil progeny emergence; $\mathrm{GD}=$ Percent grain damage; MDP = median development period; DIS = Dobie index of susceptibility, $\mathrm{PWM}=$ parental weevil mortality; GxE = Genotype by Environment interactions 
emergence (FWE), and median development period (MDP) indicated that genotypes and environments were significant $(\mathrm{P}<0.001)$; while for parental weevil mortality (PWM), all effects and interactions were not significant $(\mathrm{P}>0.05)$. Genotype $\mathrm{x}$ environment interactions were significant for grain damage $(\mathrm{P}<0.001)$ and DIS $(\mathrm{P}<0.05)$; whereas they were not significant $(\mathrm{P}>0.05)$ for $\mathrm{F}_{1}$ weevil emergence, median development period and parental weevil mortality.

Results for the mean response of the 20 most weevil resistant and five most susceptible inbred lines, together with the two resistant checks as revealed by four weevil screening parameters are shown in Table 2. There were significant $(\mathrm{P}<0.05)$ differences among inbred lines for each of the four weevil screening parameters. Inbred lines MV13, MV21, MV23, MV31, MV63, MV75, MV102, MV105, MV142, MV154, MV157, MV170, and MV175 were grouped among the 20 most weevil resistant inbred lines by the four susceptibility parameters. The number of $F_{1}$ weevil progenies that emerged from the grain of the 20 most resistant inbred lines were not significantly ( $P>0.05)$ different from those which emerged from the resistant checks. Inbred lines MV21, MV23, MV75, MV102, MV142, MV157, MV165, and MV170 supported fewer $F_{1}$ weevil progenies, but were not significantly different from those of the resistant checks. The smallest number of $F_{1}$ weevil progeny emergence was 5.67, exhibited by inbred line MV165; while the largest was 151.50 exhibited by inbred line MV44. The two most susceptible genotypes, MV44 and MV83, supported a significantly $(\mathrm{P}<0.05)$ higher number of $\mathrm{F}_{1}$ weevil progenies than the susceptible checks.
Regarding grain damage, the least grain damage was $8.20 \%$, exhibited in inbred line MV170; while the highest damage was $23.74 \%$, encountered by inbred line MV9 (Table 2). The responses of the 14 most resistant inbred lines, namely MV13, MV21, MV23, MV31, MV63, MV75, MV102, MV105, MV142, MV154, MV157, MV165, MV170, and MV175 did not significantly $(\mathrm{P}>0.05)$ differ from that of the resistant checks. Also, the damage exhibited in the three most susceptible inbred lines did not significantly differ from that of the susceptible checks.

For the median development period, it ranged from 29.33 days in a susceptible inbred line MV2, to 52 days in the resistant inbred line MV170. All the 20 most weevil resistant inbred lines (Table 2), were not significantly $(\mathrm{P}>0.05)$ different from the resistant checks. Inbred lines MV21, MV23, MV31, MV63, MV75, MV154, MV170, and MV178 exhibited long median development periods, and were not significantly $(\mathrm{P}>0.05)$ different from the resistant checks. Similarly, the five most susceptible inbred lines, (MV2, MV41, MV90, MV138, and MV155) were also not significantly $(\mathrm{P}>0.05)$ different from the susceptible checks.

As regards to Dobie's index of susceptibility, the lowest value was 3.20, manifested in inbred line MV170; while the largest index was 15.61, exhibited by inbred line MV41. Values for inbred lines MV13, MV21, MV23, MV31, MV63, MV75, MV102, MV105, MV142, MV154, MV157, MV170, and MV175 were not significantly $(\mathrm{P}>0.05)$ different from those of the resistant checks. In the same regard, values for the five most susceptible inbred lines MV9, MV33, MV41, MV78, and MV138 were not significantly different from those of the susceptible checks. 
Genotypic variation for maize weevil resistance

Table 2. Mean responses of the 20 top weevil resistant and 5 most susceptible inbred lines compared to the resistant and susceptible checks, as revealed by the four weevil screening parameters

\begin{tabular}{|c|c|c|c|c|c|c|c|c|}
\hline Checks & Genotype & FWE & Genotype & GD & Genotype & MDP & Genotype & DIS \\
\hline \multirow[t]{20}{*}{ Top 20} & MV165 & 5.7 & MV170 & 8.2 & MV31 & 52.0 & M170 & 3.2 \\
\hline & MV170 & 5.8 & MV75 & 8.4 & MV170 & 52.0 & MV75 & 3.4 \\
\hline & MV75 & 6.7 & MV157 & 8.7 & MV21 & 51.7 & MV157 & 3.7 \\
\hline & MV102 & 7.0 & MV21 & 8.7 & MV154 & 51.3 & MV21 & 3.7 \\
\hline & MV142 & 7.0 & MV142 & 8.8 & MV23 & 51.0 & MV142 & 3.8 \\
\hline & MV157 & 7.0 & MV102 & 8.8 & MV63 & 50.7 & MV102 & 3.8 \\
\hline & MV21 & 7.3 & MV23 & 9.0 & MV75 & 50.3 & MV23 & 4.0 \\
\hline & MV23 & 7.8 & MV31 & 9.0 & MV178 & 50.2 & MV31 & 4.0 \\
\hline & MV63 & 8.0 & MV63 & 9.0 & MV157 & 49.7 & MV63 & 4.0 \\
\hline & MV154 & 8.2 & MV154 & 9.1 & MV102 & 49.3 & MV154 & 4.1 \\
\hline & MV31 & 9.5 & MV165 & 9.1 & MV169 & 49.3 & MV165 & 4.1 \\
\hline & MV105 & 9.7 & MV105 & 9.4 & MV105 & 49.0 & MV105 & 4.4 \\
\hline & MV175 & 10.5 & MV13 & 9.5 & MV144 & 48.8 & MV13 & 4.5 \\
\hline & MV13 & 10.7 & MV175 & 9.8 & MV177 & 48.3 & MV175 & 4.8 \\
\hline & MV12 & 11.2 & MV163 & 10.6 & MV182 & 48.3 & MV163 & 5.6 \\
\hline & MV163 & 13.5 & MV169 & 10.7 & MV174 & 48.2 & MV169 & 5.7 \\
\hline & MV159 & 14.0 & MV159 & 10.8 & MV6 & 48.0 & MV159 & 5.8 \\
\hline & MV19 & 14.2 & MV177 & 10.8 & MV13 & 48.0 & MV177 & 5.8 \\
\hline & MV168 & 15.0 & MV168 & 10.9 & MV142 & 47.7 & MV168 & 5.9 \\
\hline & MV164 & 16.7 & MV174 & 10.9 & MV175 & 47.3 & MV174 & 5.9 \\
\hline Resistant & $\mathrm{RC} 182$ & 8.0 & $\mathrm{RC} 182$ & 8.2 & $\mathrm{RC} 182$ & 50.0 & $\mathrm{RC} 182$ & 3.7 \\
\hline Checks & RC181 & 7.2 & $\mathrm{RC} 181$ & 9.3 & $\mathrm{RC} 181$ & 48.3 & $\mathrm{RC} 181$ & 3.2 \\
\hline \multicolumn{9}{|l|}{ Bottom 5} \\
\hline & MV88 & 120.2 & MV83 & 22.2 & MV155 & 30.0 & MV78 & 14.8 \\
\hline & MV104 & 130.7 & MV100 & 22.3 & MV41 & 29.7 & MV33 & 14.8 \\
\hline & MV33 & 132.8 & MV44 & 22.8 & MV90 & 29.7 & MV138 & 15.1 \\
\hline & MV83 & 143.7 & MV33 & 23.2 & MV138 & 29.7 & MV9 & 15.4 \\
\hline & MV44 & 151.5 & MV9 & 23.7 & MV2 & 29.3 & MV41 & 15.6 \\
\hline Susceptible & SC183 & 113.3 & SC183 & 24.9 & SC183 & 32.3 & SC183 & 15.5 \\
\hline Checks & SC184 & 132.7 & SC184 & 23.0 & SC184 & 30.5 & SC184 & 14.1 \\
\hline $\operatorname{LSD}(0.05 \%)$ & & 30.0 & & 2.3 & & 4.2 & & 1.5 \\
\hline Trial mean & & 65.7 & & 16.4 & & 37.8 & & 10.8 \\
\hline
\end{tabular}

$\mathrm{FWE}=\mathrm{F}_{1}$ weevil progeny emergence, $\mathrm{GD}=$ Percent grain damage, $\mathrm{MDP}=$ median development period, and DIS $=$ Dobie index of susceptibility 
Frequency distribution of inbred lines checks, and, hence, were categorised in into different weevil response classes the resistant class $\left(0.0-30.0 \mathrm{~F}_{1}\right.$ weevil Results for the distribution of the 180 progenies). Forty inbred lines were inbred lines into different response classes grouped in the moderately resistant class as grouped by $F_{1}$ weevil progeny $\left(30.1-60.0, F_{1}\right.$ weevil progenies), 60 emergence, grain damage, median inbred lines were grouped in the development period, and Dobie's index of moderately susceptible class (60.1-90.0); susceptibility are shown in Figure 1. The while 46 inbred lines were grouped in the results of the $F_{1}$ weevil progeny susceptible class $(>90.1)$.

emergence (Fig. 1a) showed that 35 The distribution of inbred lines into the inbred lines were not significantly four response categories exhibited a $(\mathrm{P}>0.05)$ different from the resistant normal distribution trend, with the majority
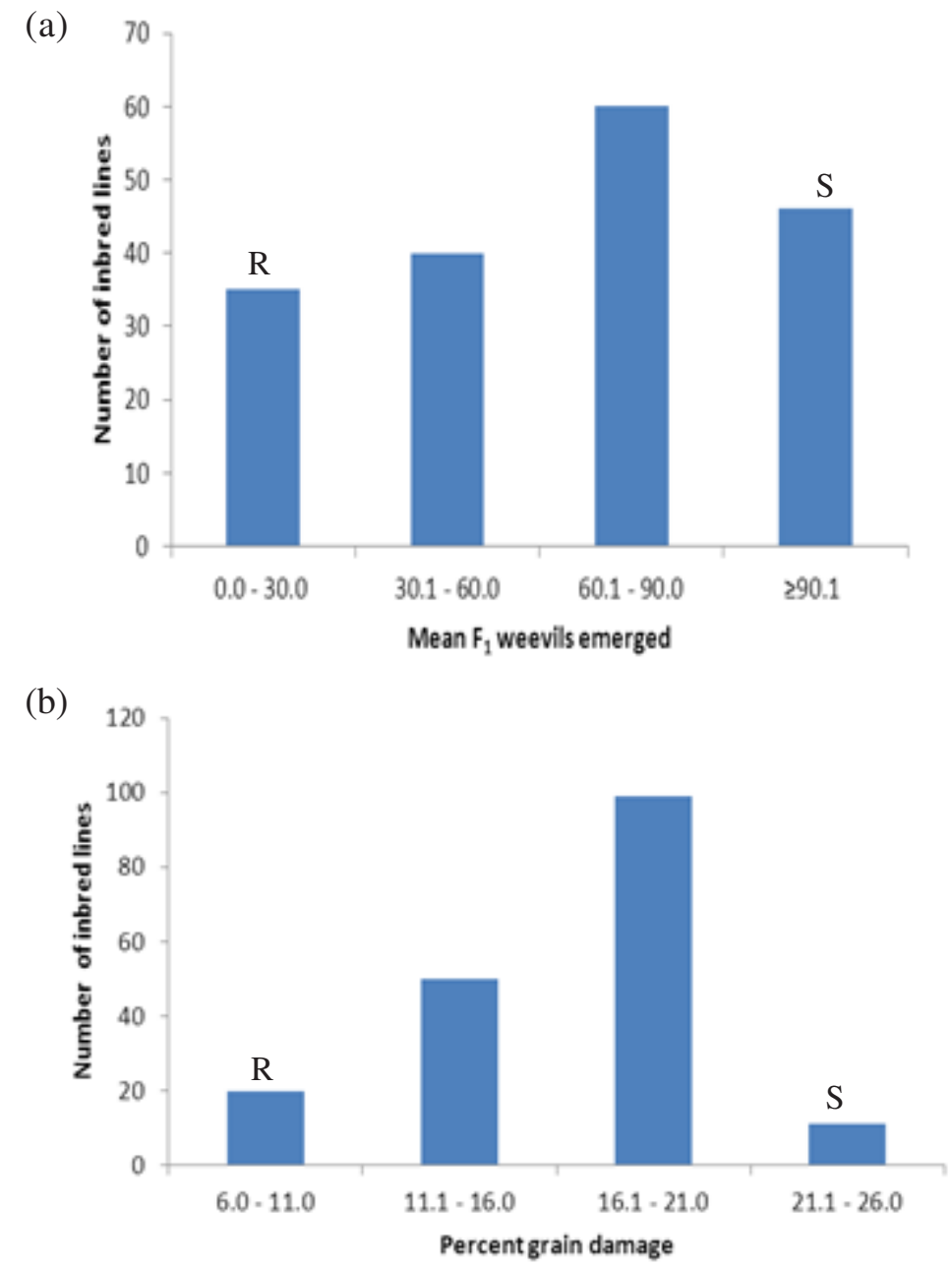

Figure 1. Frequency distribution of 180 inbred lines into different weevil response classes as grouped by the four weevil screening parameters: a) $F_{1}$ weevil progeny emergence; $b$ ) percent grain damage. 
(c)

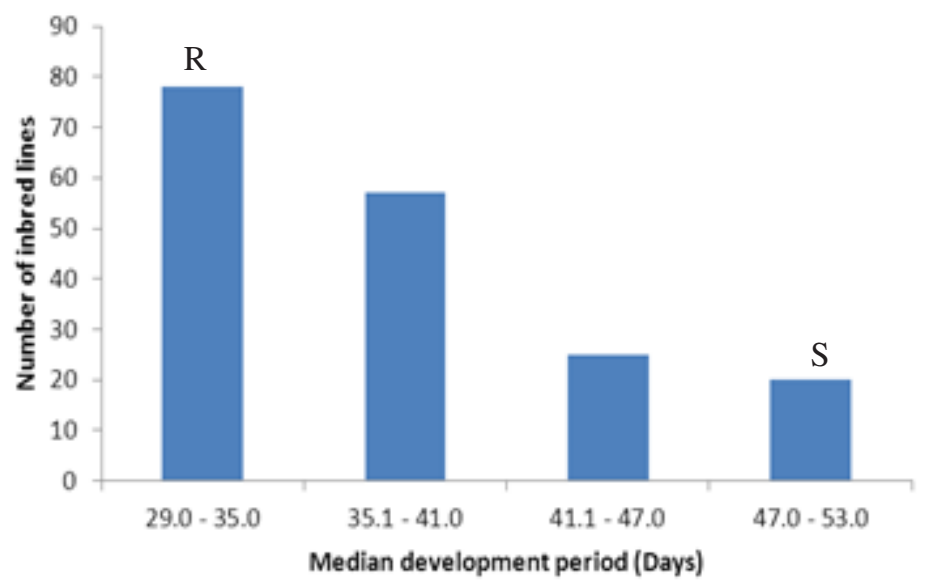

(d)

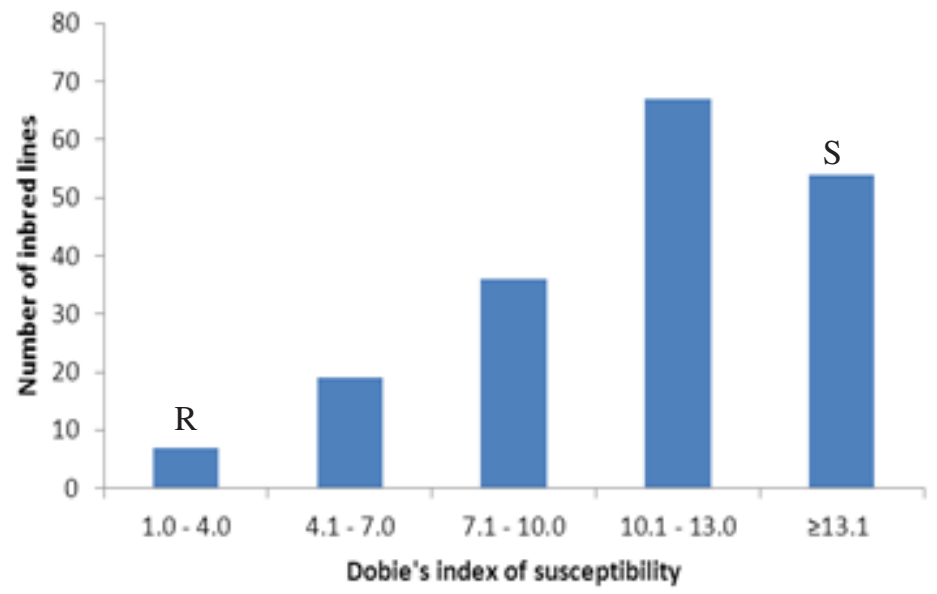

Figure 1. Frequency distribution of 180 inbred lines into different weevil response classes as grouped by the four weevil screening parameters: c) median development period; d) Dobie index of susceptibility. $R$ and $S$ represent the classes where the resistant $(R)$ and susceptible (S) checks fell, respectively.

of inbred lines (60) being in the moderately susceptible class under which the trials mean (65.7 weevils) fell. The resistant and susceptible checks were noted to fall under their respective, resistant and susceptible classes, with few inbred lines falling in the moderately resistant and moderately susceptible classes.

Results of grain damage (Fig. 1b) exhibited the same trend as that of $F_{1}$ weevil progeny emergence. Fourteen inbred lines encountered grain damage that was not significantly different from the resistant checks, and were hence grouped in the resistant class $(6.0-11.0 \%$ damage). Fifty six inbred lines were grouped in the moderately resistant class $(11.1-16.0 \%), 99$ were grouped in the moderately susceptible class (16.1 $21.0 \%$ ), while 11 were grouped in the 
susceptible class $(21.1-26.0 \%)$. The distribution of inbred lines exhibited a trend close to a normal distribution curve, with the majority of inbred lines falling under the moderately resistant and moderately susceptible classes under which the trial mean $(16.42 \%$ damage) fell. The resistant and susceptible checks fell under the resistant- and susceptible-classes, respectively.

For the median development period (Fig. 1c), 20 inbred lines were grouped in the resistant class ( $47.0-53.0$ days), 25 in the moderately resistant class (41.1 47.0 days), 57 in the moderately susceptible class ( $35.1-41.0$ days); while 78 were grouped in the susceptible class (29.0 - 35.0 days). Here, the distribution of inbred lines was skewed to the susceptible side of the curve.

Results of the Dobie's index of susceptibility (Fig. 1d), exhibited an inverse trend to that of the MDP, with the inbred lines that exhibited longer MDPs displaying smaller indices; while those with shorter MDPs displayed larger indices. Consequently, eight inbred lines with indices $1.0-4.0$ were categorised as resistant, 19 inbred as moderately resistant $(4.1-7.0) ; 34$ as moderately susceptible
$(7.1-10.0) ; 65$ as susceptible $(10.01-$ 13.0); while 54 inbred lines were categorized as highly susceptible. The distribution of inbred lines into the various response classes generally followed a normal distribution curve, with a slight tendency of skewing to the susceptible side of the curve.

Overall, only $4.4 \%$ of the total samples of 180 inbred lines displayed resistance to the maize weevil, and were hence categorized in the resistant class. Inbred lines fitted in the moderately resistant class constituted 10.6\%; inbred lines that fell under moderately susceptible constituted $18.9 \%$ those that were grouped as susceptible constituted $36.1 \%$, while $30 \%$ of the inbred lines were categorised as highly susceptible (Table 3 ).

\section{Heritability estimates}

The narrow sense heritability estimates for $F_{1}$ weevil progeny emergence, grain damage, median development period, Dobie's index of susceptibility and grain yield are shown in Table 4. The genetic variances for $F_{1}$ weevil progeny emergence (FWE), grain damage (GD), median development period (MDP) and Dobie index of susceptibility (DIS) were

Table 3. Distribution of inbred lines from eastern and Southern Africa into different weevil response classes as categorised by Dobie's index of susceptibility

\begin{tabular}{|c|c|c|c|c|c|c|c|c|}
\hline \multirow[t]{2}{*}{ Class } & \multicolumn{2}{|c|}{ Uganda } & \multicolumn{2}{|c|}{ CIMMYT-Kenya } & \multicolumn{2}{|c|}{ South Africa } & \multicolumn{2}{|c|}{ Overall total } \\
\hline & No. & $\%$ & No. & $\%$ & No. & $\%$ & No. & $\%$ \\
\hline $1.0-4.0$ & 4 & 7.8 & 2 & 3.6 & 2 & 2.7 & 8 & 4.4 \\
\hline $4.1-7.0$ & 13 & 25.5 & 5 & 9.1 & 1 & 1.4 & 19 & 10.6 \\
\hline $7.1-10.0$ & 16 & 31.4 & 13 & 23.6 & 5 & 6.8 & 34 & 18.9 \\
\hline $10.1-13.0$ & 14 & 27.5 & 15 & 27.3 & 36 & 48.6 & 65 & 36.1 \\
\hline e"13.1 & 4 & 7.8 & 20 & 36.4 & 30 & 40.5 & 54 & 30.0 \\
\hline Total & 51 & & 55 & & 74 & & 180 & \\
\hline
\end{tabular}




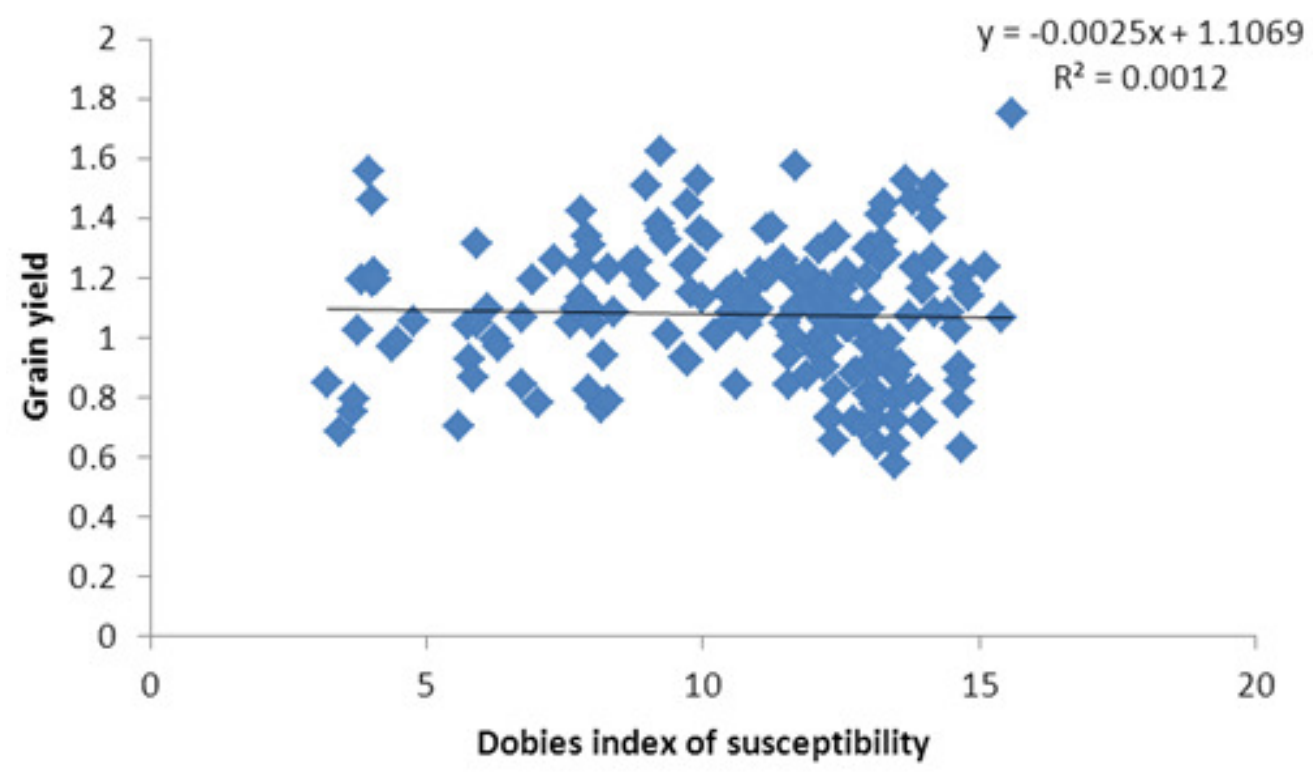

Figure 2. Scatter plot for the relationship between Dobie's index of susceptibility and grain yield.

Table 4. Narrow sense heritability estimates for the four weevil screening parameters, grain yield and three foliar diseases exhibited in the 180 inbred lines

\begin{tabular}{llllll}
\hline Variance component & \multicolumn{5}{c}{ Means \pm SE } \\
\cline { 2 - 6 } & \multicolumn{1}{c}{ FWE } & GD & MDP & DIS & Yield \\
\hline $\mathrm{Vg}$ & $1032.0 \pm 121.6$ & $11.9 \pm 1.34$ & $30.56 \pm 3.47$ & $8.81 \pm 0.97$ & $0.03 \pm 0.01$ \\
$\mathrm{Ve}$ & $700.10 \pm 33.1$ & $4.63 \pm 0.22$ & $13.54 \pm 0.64$ & $1.94 \pm 0.09$ & $0.12 \pm 0.01$ \\
$\mathrm{~h}^{2}$ & $89.84 \pm$ & $93.92 \pm$ & $93.12 \pm$ & $96.45 \pm$ & $58.28 \pm$ \\
\hline
\end{tabular}

$\mathrm{FWE}=\mathrm{F}_{1}$ weevil progeny emergence, $\mathrm{GD}=$ Percent grain damage, $\mathrm{MDP}=$ median development period, and DIS = Dobie index of susceptibility

large relative to their respective environmental variances. Consequently, they all portrayed high heritability estimates above $89 \%$.

\section{Relationship between weevil resistance and grain yield}

The regression between Dobie's index of susceptibility and grain yield gives a scatter plot in Figure 2. The results show a parallel distribution of scatter points along the $\mathrm{X}$ - axis with a low coefficient of determination $\left(\mathrm{R}^{2}\right)$ value.

\section{Discussion}

Genotype response to weevil infestation The significant environments portrayed by the mean squares for the four weevil screening parameters indicate that the differences among the inbred lines were partly attributed to the differences in the 
two seasons under which the trial was conducted (Li et al., 1998). On the other hand, the significant genotype mean squares demonstrated under the four weevil screening parameters indicate that the 180 inbred lines exhibited genetic variations for weevil resistance. The existence of genetic variations for weevil resistance in maize germplasm from eastern and southern African region shows a great potential of new sources of weevil resistance that can be exploited in the subsequent development of weevil resistant maize cultivars. Genetic variations for weevil resistance have been observed in maize germplasm, including hybrids and open pollinated varieties (Mwololo et al., 2012).

The significant genotype $\mathrm{x}$ environment interactions for grain damage and Dobie's index of susceptibility, suggests differences in genotype response to weevil infestations between seasons. The differences in genotype response could be attributed to the differences in grain characteristics, that might be manifested as a result of changes in the environment. For example, grain characteristics like texture, and to some extent, kernel hardness might be affected by drought, which may limit grain filling and this would consequently affect grain texture and/or hardness. Manifestation of significant genotype $\mathrm{x}$ environment interactions is consistent with observations by Tipping et al. (1989).

The mean square results suggested that the four weevil screening parameters namely: $F_{1}$ weevil progeny emergence, grain damage, median development period and Dobie's index of susceptibility were able to discriminate inbred lines based on their variations in weevil susceptibility (Abebe et al., 2009). However, parental weevil mortality, as a weevil susceptibility parameter, did not detect significant
$(\mathrm{P}>0.05)$ differences in weevil resistance among the 180 test inbred lines. This implied that parental weevil mortality was not a suitable parameter for discriminating maize cultivars for weevil resistance.

\section{Inbred distribution into different weevil resistance classes}

The distribution of inbred lines into the different response classes exhibited a "generally" continous (normal) distribution (Fig. 1 a, b, and d), implying that weevil resistance is controlled by quantitative genes; hence breeding procedures that exploit polygenes and quantitative trait loci QTLs are recommended in breeding for weevil resistance. It was observed that in the resistant inbred lines, fewer $F_{1}$ weevil progenies emerged, and these took a longer period to emerge, as opposed to the susceptible inbred lines which encountered more $F_{1}$ weevil progeny emergence in a shorter period.

The fewer $F_{1}$ weevil progenies and longer MDP in the resistant inbred lines could be explained by the higher quantities of biochemical compounds such as phenolics and peroxidases. The biochemical compounds ought to lower weevils' reproduction rates as a result of antibiosis exhibited in resistant maize genotypes. This phenomenon is in line with findings by Derera et al. (2001) and García-Lara et al. (2009), who reported high levels of biochemical compounds and antibiosis in weevil resistant maize genotypes.

The results from the four weevil screening parameters, grouped the same inbred lines in similar response classes (Fig. 1). This implies that the four weevil screening parameters were consistent in discriminating the inbred lines. Inbred lines MV13, MV21, MV23, MV31, MV63, MV75, MV102, MV105, MV142, 
MV154, MV157, MV170, and MV175 were grouped among the 20 most weevil resistant inbred lines by the four susceptibility parameters used in the study. Weevil resistant inbred lines that were not significantly different from the resistant checks are potential parents for development of weevil resistant maize cultivars. Thus, inbred lines MV142, MV154, MV157, and MV170 from Uganda; inbred lines MV21 and MV23 from CIMMYT-Kenya; and inbred lines MV75 and MV102 from South Africa that were consistently grouped in the resistant categories and portrayed good performances comparable to the resistant check are potential parents for breeding new varieties with weevil resistance.

The existence of weevil resistant inbred lines among maize germplasm from different sources demonstrates high genetic variability for weevil resistance. This suggests an opportunity for broadening the genetic base for weevil resistance, since the inbred lines were from three different geographical areas, which may imply differences in the sources of genetic resistance. However, further tests involving molecular markers are recommended to verify whether these materials are genetically different.

\section{Heritability}

The large heritability values (above 89\%) for weevil resistance exhibited by the four weevil screening parameters (Table 4), implies that a greater proportion of the phenotypic expression of the inbred lines was contributed by the genotypic variance. Thus, there was minimal environmental variance that influenced the four weevil screening parameters/grain resistance against weevil infestations in the inbred lines. The large heritability manifested in the four parameters, suggests that selection would be effective in improving the germplasm towards weevil resistance. The large heritability values for the susceptibility parameters are consistent with García-Lara et al. (2010) who reported up to $79 \%$ heritability of weevil resistant molecular traits. Grain yield exhibited moderate heritability indicating that selection would be quite effective towards improving grain yield in the germplasm.

Regarding the association between Dobie's index of susceptibility and grain yield (Fig. 2 ), the small $\mathrm{R}^{2}$ value $(<1.0 \%)$ and the parallel distribution of coordinates along the $\mathrm{X}$-axis manifested in the scatter plots, indicates insignificant associations between the two traits. This suggests that improvement of the study germplasm for weevil resistance would not necessarily compromise grain yield, thus suggesting that each of the two traits can be improved independently without affecting one another.

\section{Acknowledgement}

This study was conducted with funding from Alliance for Green Revolution in Africa (AGRA). The research facilities and part of the germplasm were provided by the National Crops Resources Research Institute (NaCRRI). The International Maize and Wheat Improvement Centre (CIMMYT) and University of KwaZulu Natal, Republic of South Africa, provided additional germplasm.

\section{References}

Abebe, F., Tefera, T., Mugo, S., Beyene, Y. and Vidal, S. 2009. Resistance of maize varieties to the maize weevil Sitophilus zeamais (Motsch.) 
(Coleoptera: Curculionidae). African Journal of Biotechnology 8: 59375943.

Dari, S., Pixley, K.V. and Setimela, P. 2010. Resistance of early generation maize inbred lines and their hybrids to maize weevil Sitophilus zeamais (Motschulsky). Crop Science 50: 1310-1317.

Demisse, G., Tafera, T. and Tadesse, A., 2008. Importance of husk covering on field infestation of maize by Sitophilus zeamais Motsch (Coleoptera: Curculionidea) at Bako, Western Ethiopia. African Journal of Biotechnology 7: 3774-3379.

Derera, J., Pixley, K.V. and Giga, D. 2001. Resistance of maize to the maize weevil. I. Antibiosis. African Crop Science Journal 9: 431- 440.

Derera, J., Tongoona, P., Vivek, B.S. and Laing, M.D. 2007. Gene action controlling grain yield and secondary traits in southern African maize hybrids under drought and non-drought environments. Euphytica 162: 411422.

Dobie, P. 1974. The laboratory assessment of the inherent susceptibility of maize varieties to postharvest infestation by Sitophilus zeamais. Journal of Stored Products Research 10: 183197.

Dobie, P. 1977. The contribution of the tropical stored products center to the study of insect resistance in stored maize. Tropical Stored Products Information 34: 7-22.

García-Lara, S., Burt, A.J., Arnason, J.T. and Bergvinson, D. 2010. QTL mapping of tropical maize grain components associated with maize weevil resistance. Crop Science 50: 815-825.
García-Lara, S., Khairallah, M., Vargas, M. and Bergvinson, D. 2009. Mapping of QTL associated with maize weevil resistance in tropical maize. Crop Science 49: 139-149.

Hallauer, A.R. and Miranda, J.B., 1988. Quantitative genetics in maize breeding. Iowa State University Press. Ames, IA, USA.

Li, R., Kang, M.S, Moreno, O.J. and Pollak, L.M. 1998. Genetic variability in exotic $\mathrm{x}$ adapted maize (Zea mays L.) germplasm for resistance to maize weevil. Plant Genetic Resources Newsletter 114: 22-25.

Makumbi, D., Betrán, J.F., Bänziger, M. and Ribaut, J.M., 2011. Combining ability, heterosis and genetic diversity in tropical maize (Zea mays L.) under stress and non-stress conditions. Euphytica 180: 143-162.

Mwololo, J., Mugo, S., Okori, P., Tefera, T. and Munyiri, S. 2010. Genetic diversity for resistance to larger grain borer in maize hybrids and open pollinated varieties in Kenya. Second RUFORUM Biennial Meeting, Entebbe, Uganda, 20 - 24 September.

Mwololo, J., Mugo, S., Okori, P., Tefera, T., Otim, M. and Munyiri, S.W. 2012. Sources of resistance to the maize weevil Sitophilus zeamais in tropical maize. Journal of Agricultural Science 4: 206-215.

Payne, R.W., Murray, D.A., Harding, S.A., Baird, D.B. and Soutar, D.M., 2011. An introduction to Genstat for windows (14th edition). VSN international, Hemel Hempstead, UK.

Siwale, J., MacRobert, J. and Lungu, D., 2009. Comparative resistance of improved maize genotypes and landraces to maize weevil. African Crop Science Journal 17: 1-16. 
Tipping, P.W., Cornelius, P.L., Legg, D.E., Poneleit, C.G. and Rodriguez, J.G. 1989. Inheritance of resistance in whole kernel maize to oviposition by the maize weevil (Coleoptera: Curculionidae). Entomological Society of America 82: 1466-1469.

Tollenaar, M. and Lee, E.A. 2006. Dissection of physiological processes underlying grain yield in maize by examining genetic improvement and heterosis. Maydica 51: 399-408.

Vivek, B.S., Odong, O., Njuguna, J., Imanywoha, J., Bigirwa, G. and Diallo, A. 2010. Diallel analysis of grain yield and resistance to seven diseases of 12 African maize (Zea mays L.) inbred lines. Euphytica 172: 329-340.

Widstrom, N.W., McMillian, W.W., Redlinger, L.M. and Wiser, W.J., 1983. Dent corn inbred sources of resistance to the maize weevil (Coleoptera: Curculionidae). Journal of Economic Entomology 76: 31-33.

Widstrom, N.W., Redlinger, L.M. and Wiser, W.J. 1972. Appraisal of methods for measuring corn kernel resistance to Sitophilus zeamais. Journal of Economic Entomology 65: 790-792. 